\title{
Angleterre : premiers résultats controversés de l'état des lieux sur l'enseignement primaire
}

\section{Maroussia Raveaud}

\section{OpenEdition}

1 Journals

Édition électronique

URL : http://journals.openedition.org/ries/382

DOI : $10.4000 /$ ries.382

ISSN : 2261-4265

Éditeur

Centre international d'études pédagogiques

\section{Édition imprimée}

Date de publication : 1 septembre 2008

Pagination : 13-16

ISBN : 978-2-85420-573-2

ISSN : $1254-4590$

Référence électronique

Maroussia Raveaud, «Angleterre : premiers résultats controversés de l'état des lieux sur

l'enseignement primaire », Revue internationale d'éducation de Sèvres [En ligne], 48 | septembre 2008 , mis en ligne le 27 juin 2011, consulté le 26 avril 2021. URL : http://journals.openedition.org/ries/382 DOI : https://doi.org/10.4000/ries.382

Ce document a été généré automatiquement le 26 avril 2021

(c) Tous droits réservés 


\title{
Angleterre : premiers résultats controversés de l'état des lieux sur l'enseignement primaire
}

\author{
Maroussia Raveaud
}

\section{Une commission d'enquête farouchement indépendante}

1 Alors que l'Unicef a placé le Royaume-Uni en queue d'une classification internationale du bien-être des enfants ${ }^{1}$, une grande commission d'enquête sur l'enseignement primaire, The Primary Review, est sur le point d'aboutir. Ses objectifs et son mode de fonctionnement ont été présentés dans un précédent numéro² ${ }^{2}$ Fin mai 2008, 29 des 31 rapports de recherche ont été publiés, fortement critiques de deux décennies de réformes intensives du système primaire anglais. Reste à venir la synthèse qui sera effectuée sous la direction du professeur Robin Alexander, ainsi que le rapport de recherche qu'il co-signera avec Maurice Galton sur l'enseignement à l'école primaire, mais l'impact des travaux de la Commission se fait déjà largement ressentir par les controverses qu'elle soulève et les réactions gouvernementales qu'elle provoque.

Rappelons que l'initiative n'a rien d'officiel et n'est pas d'origine gouvernementale : la Commission, basée à l'Université de Cambridge, s'appuie sur des rapports de recherche rédigés par soixante-dix universitaires, et son financement provient de la fondation Esmée Fairbairn. Son indépendance lui permet une totale liberté dans le choix des sujets à traiter et un ton critique à l'égard des politiques officielles.

Le rythme et l'ampleur des réformes débutées sous le gouvernement de Margaret Thatcher et étendues sous celui de Tony Blair donnent inévitablement à cet état des lieux un caractère d'audit du gouvernement, que ce dernier n'avait en rien sollicité et pour lequel il n'a guère témoigné d'enthousiasme à l'origine. Toutefois, la démarche a reçu l'appui des autres grands acteurs éducatifs du pays, dont la commission parlementaire sur l'éducation, le corps d'inspection, les syndicats enseignants, les 
autorités locales et des chercheurs reconnus; et le ministère a promis sa « coopération ».

\section{Un état des lieux national aux enjeux globaux}

Outre l'étude des politiques gouvernementales, l'enquête couvre un large spectre depuis les missions et valeurs de l'école jusqu'à son adéquation aux nouvelles réalités économiques et sociales en passant par les pratiques pédagogiques, l'évaluation, la profession enseignante, le rôle des parents, les bâtiments scolaires, ou encore l'impact de la mondialisation ${ }^{3}$.

5 Inévitablement, certains enjeux traités intéressent au premier chef l'Angleterre, tels que le bilan des réformes conservatrices et néo-travaillistes des vingt dernières années. Toutefois, de nombreux enjeux étudiés sont à portée plus large, par exemple l'impact des évaluations nationales sur les enseignements, sur la progression des élèves et sur la gouvernance, ou encore la recherche d'un équilibre entre la reconnaissance de la diversité des populations scolaires et la promotion de cohésion sociale et de valeurs partagées. Les comparaisons internationales sont ponctuellement mobilisées dans plusieurs rapports de recherche, et cinq sont à vocation comparative. Ainsi, des études de cas sur l'Inde et la Chine sont proposées pour illustrer la façon dont la réforme de ces systèmes éducatifs articule besoins nationaux et tendances internationales. La France est mobilisée, aux côtés de la Norvège, du Japon et de dix-huit autres pays pour analyser les politiques en matière de programmes d'enseignement et d'évaluations nationales. Ces comparaisons internationales sont à mettre en rapport avec l'objectif avancé par la commission de replacer des évolutions à l'échelle locale et nationale dans le contexte de la mondialisation.

\section{Les conclusions : " théorie étatique de l'apprentissage » et « stalinisme de marché »}

6 Notons que les conclusions disponibles à ce stade sont celles des auteurs des rapports et non encore la synthèse finale de la Primary Review, et que les rapports de recherche ont pour mission de faire l'état des lieux des travaux existants plutôt que de présenter le point de vue des auteurs. Au-delà de différences d'approches et des sujets traités, le degré de consensus est remarquable sur des points clés :

7 - les réformes, débutées sous les conservateurs, puis multipliées et accélérées sous le gouvernement néo-travailliste, n'ont pas produit de retombées à la hauteur des investissements et des effets anticipés par le gouvernement ;

8 - un interventionnisme étatique aussi bien direct - portant sur les programmes d'enseignement et les pratiques pédagogiques - qu'indirect - par les standards et l'inspection - conduit certes à l'amélioration des résultats tels que mesurés par les tests nationaux, mais au détriment de la diversité des apprentissages, de la richesse de l'expérience scolaire des élèves, et de l'autonomie des enseignants ;

9 - des initiatives gouvernementales relevant de cadres idéologiques potentiellement contradictoires conduisent à des tensions au niveau des priorités politiques et du rôle attendu des acteurs éducatifs. Si le ministère avance une conception large et riche des savoirs et compétences dans ses programmes, la restriction des évaluations nationales 
aux disciplines de base (langue, mathématiques et sciences) limite de fait l'enseignement pratiqué dans les écoles. De même, l'objectif de résorption des inégalités entre en conflit avec la dynamique néo-libérale du marché et la concurrence éducative qui conduisent à l'accroissement des inégalités et de la ségrégation sociale et ethnique.

\section{Un accueil enthousiaste, sauf au ministère}

10 La presse spécialisée et les grands quotidiens nationaux ont relayé la publication des rapports de recherche avec des manchettes marquantes : «Perte de l'enfance du fait de la pression des tests ", "Ne nous faites pas bachoter, demandent les enfants ", "Les écoles primaires ont empiré $»^{4}$. Les syndicats, notamment le NUT, principal syndicat de l'enseignement primaire, applaudissent les conclusions et appellent le gouvernement à les prendre en considération. Le parti libéral démocrate abonde dans le sens d'un interventionnisme excessif et néfaste, tandis que les conservateurs promettent de rendre plus de liberté aux établissements. Même le directeur de l'inspection (Qualifications and Curriculum Authority, QCA) a soutenu l'appel à un système d'évaluation par échantillonnage à la place de l'actuel test de cohortes entières, allant par là à l'encontre des positions gouvernementales.

11 Les réactions du ministère ont été moins enthousiastes et ont pris deux formes. La première consiste à défendre les positions officielles. Face aux critiques sévères portant sur l'effet des évaluations nationales sur le stress des élèves et l'appauvrissement des enseignements, le ministère a démenti l'idée d'une culture de l'évaluation, en réaffirmant que sa priorité était que tout élève quitte le système éducatif en ayant acquis les bases attendues dans les trois disciplines fondamentales ${ }^{5}$. Cette défense passe également par le discrédit jeté sur les résultats de la Primary Review : à la critique d'un interventionnisme pédagogique conduisant à une "théorie étatique de l'apprentissage ", la porte-parole du ministère a répondu qu'il s'agissait d'une "recherche recyclée, biaisée et périmée » ${ }^{6}$. Le secrétaire d'État délégué à l'enfance ajoute : «Je ne vais pas m'excuser de promouvoir ce qu'attendent les parents même si ça ne plaît pas à ces chercheurs, qui s'appuient sur de la recherche souvent datée $»^{7}$.

12 La seconde réaction a consisté à annoncer des mesures en amont de la publication des rapports. C'est ainsi que le ministère a annoncé de nouvelles procédures d'appel le 17 janvier 2008, à la veille de la publication d'un rapport condamnant les effets discriminatoires et inégalitaires des systèmes d'admission actuellement pratiqués par les établissements. Si l'initiative ministérielle ne couvre de fait pas l'intégralité des enjeux soulevés dans le rapport de recherche, notamment la question des stratégies résidentielles des familles plus fortunées, la position officielle en réaction au rapport a été qu'aucune mesure supplémentaire ne serait nécessaire.

De même, l'annonce en janvier 2008 d'une commission officielle chargée de réviser les programmes de l'enseignement primaire a été interprétée comme une stratégie pour marginaliser la commission indépendante qu'est la Primary Review et le rapport de recherche initialement prévu sur les programmes a été annulé, ses auteurs ayant subi des pressions. La mission de la commission gouvernementale recoupe en partie le travail effectué par l'équipe de Robin Alexander, à deux restrictions près, et de taille : l'objectif est imposé, à savoir l'amélioration des résultats, et le régime d'évaluations et de tests est fermement placé hors de son champ de compétence. C'est en fonction des 
résultats de cette commission, dont le rapport final est attendu pour mars 2009, que les programmes de l'enseignement primaire seront révisés à compter de la rentrée 2011. Le travail en cours permet au ministère d'éviter de répondre directement aux conclusions de la Primary Review.

\section{Une nouvelle « bible » pour l'enseignement primaire?}

14 Quarante ans après le rapport Plowden, qualifié de «bible» d'une génération d'enseignants, la Primary Review a pour vocation de faire l'état des lieux dans un contexte politique, social et éducatif radicalement différent. Le ton et la nature des rapports sont très distincts. En l'absence de centralisation, Lady Plowden identifiait des «bonnes» pratiques existantes pour promouvoir les méthodes actives centrées sur l'enfant. La Primary Review intervient dans un contexte plus politisé et prend de fait un caractère d'audit des politiques gouvernementales. Par contraste avec l'optimisme de 1967, le processus actuel revêt un fort caractère critique, accentué par le choix des sujets traités, en ce sens que des initiatives bien reçues dans le monde enseignant telles que la politique Every Child Matters ${ }^{8}$ n'ont pas été placées au cœur des analyses.

La Commission doit remettre son rapport final début 2009. Y figureront sans doute des théories et préférences pédagogiques de Robin Alexander telles que l'autonomie construite et encadrée, l'apprentissage comme processus social ou encore «l'enseignement dialogique». Pour autant, la Primary Review restera probablement dans les mémoires comme un temps marquant dans le bras de fer politique et idéologique entre le gouvernement et une grande partie du monde éducatif, plutôt que comme une nouvelle vision pédagogique et sociale pour les écoles et les enfants de demain. On peut douter qu'elle ait vocation à devenir la nouvelle "bible" des enseignants, mais il est vrai que les temps ne s'y prêtent guère.

\section{NOTES}

1. Unicef, La pauvreté des enfants en perspective: Vue d'ensemble du bien-être des enfants dans les pays riches, Bilan Innocenti 7, 2007.

2. Revue internationale d'éducation de Sèvres $n^{\circ} 45$, septembre 2007.

3. Pour une liste complète des thèmes abordés, voir : http://www.primaryreview.org.uk

4. "Pressure of tests "means primary school pupils lose their childhood" ", The Times, 12/10/2007 ; "Don't teach to the test, say children ", The Guardian, 23/11/2007, " "Primary schools have got worse" ", BBC News, 29/2/2008.

5. "Our children tested to destruction", The Independent, 8/2/2008.

6. "Recycled, partial or out of date research", " "Primary schools have got worse" ", BBC News, $29 / 2 / 2008$.

7. Ed Balls (Children's Secretary) : "I am not going to apologise for delivering what parents want even if these researchers - on the basis of often old research - don't like it", "Ball rejects claims that political interference damages schooling ", The Independent, 1/3/08. 
8. Programme gouvernemental de 2004 consistant à créer un cadre politique et institutionnel au sein duquel les services pour l'enfance (éducation, culture, santé, services sociaux, justice...) coopèrent plus étroitement.

INDEX

Mots-clés : enseignement primaire, réforme de l'enseignement, système éducatif Index géographique : Angleterre, Royaume-Uni

\section{AUTEUR}

\section{MAROUSSIA RAVEAUD}

Maître de conférences, Université du Mans, France. 